\title{
PÉRIODICITÉ DES POLYNÔMES DE DIVISION SUR UNE COURBE ELLIPTIQUE
}

\author{
LAURENT DEWAGHE
}

\begin{abstract}
RÉSUMÉ. Soit $\mathbb{F}_{q}$ un corps de caractéristique différente de 2 et 3 et $E$ une courbe elliptique définie sur $\mathbb{F}_{q}$. Dans cet article, on considère la suite de valeurs $\left(\psi_{n}(P)\right)_{n \in Z}$ des polynômes de division de la courbe elliptique $\mathrm{E}$ en un point $P$ d'ordre fini de E. On montre, sans passage à la caractéristique zéro, que cette suite est périodique.
\end{abstract}

\section{Introduction}

Soit $E$ une courbe elliptique définie sur un corps fini $\mathbb{F}_{q}$ de caratéristique $p$ différente de 2 et 3 . L'ensemble des $\mathbb{F}_{q}$-points de $E$, noté $E\left(\mathbb{F}_{q}\right)$, est un groupe abélien fini. Pour $n \in \mathbb{N}$, les sous-groupes de $n$-torsion sont notés $E[n]$. Les polynômes de division $\psi_{n}$ de la courbe elliptique $E$ explicitent l'endomorphisme de $E\left(\mathbb{F}_{q}\right)$ de multiplication par $n$ et caractérisent le groupe $E[n]$. Les polynômes de division sont essentiels non seulement dans la théorie des courbes elliptiques mais aussi dans des domaines connexes comme l'étude des suites elliptiques. En 1948, Ward associe les valeurs d'une suite elliptique aux valeurs de polynômes de division, d'une courbe elliptique, en un point de torsion [5, 6]. Il montre, notamment, en utilisant la caractéristique zéro, qu'une suite elliptique modulo $p$ est périodique. La périodicité des suites elliptiques pour un module arbitraire est étudié par Ayads [1]. Il obtient un résultat analogue pour de "pseudo polynômes de division" $\hat{\psi}_{n}(P) \in \mathbb{Z}$, pour $P \in \mathbb{Q}$, proportionnels à $\psi_{n}(P)$. Bien qu'utilisant l'argument simple de la récurrence, sa démonstration reste néanmoins laborieuse et nécessite l'expression de $n P$ en fonction des polynômes de division et donc l'utilisation de la fonction de Weierstrass. Plus récemment, Silverman [3], étudie les propriétés $p$ adic des suites elliptiques. En particulier, il montre, en utilisant aussi un plongement dans $\mathbb{C}$, que la suite des polynômes de division modulo $p$ est périodique et précise "It would be interesting to find a purely finite field proof".

Dans cet article, on apporte, non seulement, une démonstration élémentaire et sans utiliser l'analyse complexe, de la périodicité modulo $p$ de la suite des polynômes de division $\left(\psi_{n}(P)\right)_{n \in Z}$ en un point $P \in E[r]$ mais on précise aussi le résultat connu à ce jour [1], [3].

La courbe elliptique $E$ est donnée par une équation projective définie par $\mathcal{F}(x, y, z)=y^{2} z-\left(x^{3}+a x z^{2}+b z^{3}\right)=0$ avec $a$ et $b$ dans $\mathbb{F}_{q}$. Le discriminant $\Delta=$ $4 a^{3}+27 b^{2}$ est non nul dans $\mathbb{F}_{q}$. On a

$E\left(\mathbb{F}_{q}\right)=\left\{[x: y: z] \in \mathbb{P}^{2}\left(\mathbb{F}_{q}\right) \mid \mathcal{F}(x, y, z)=0\right\}=\left\{(x, y) \in \mathbb{F}_{q}^{2} \mid \mathcal{F}(x, y, 1)=0\right\} \cup\left\{O_{E}\right\}$

Received by the editors January 25, 2005. Revision Received September 9, 2005.

2000 Mathematics Subject Classification. 11G07, 4H52.

Key words and phrases. Courbes elliptiques; polynômes de division; suites elliptiques. 
avec $O_{E}$ l'unique point à l'infini de la courbe et qui est l'élément neutre de la loi de groupe sur $E$.

La loi de groupe sur $E\left(\mathbb{F}_{q}\right)$ est précisée par la proposition suivante [4] :

Proposition 1. Soit $E$ une courbe elliptique sur $\mathbb{F}_{q}$, un corps fini de caractéristique différente de 2 et 3 , d'équation affine $\mathcal{F}(x, y, 1)=0$.

(1) Soit $P_{0}=\left(x_{0}, y_{0}\right) \in E$. Alors $-P_{0}=\left(x_{0},-y_{0}\right)$.

(2) Soit $P_{1}+P_{2}=P_{3}$ avec $P_{i}=\left(x_{i}, y_{i}\right) \in E$.

Si $x_{1}=x_{2}$ et $y_{1}=-y_{2}$, alors $P_{1}+P_{2}=O_{E}$.

Sinon, soit

$$
\left\{\begin{array}{ccc}
\lambda=\frac{y_{1}-y_{2}}{x_{1}-x_{2}} & \text { si } & x_{1} \neq x_{2} \\
\lambda=\frac{3 x_{1}^{2}+a}{2 y_{1}} & \text { si } & x_{1}=x_{2}
\end{array}\right.
$$

alors $x_{3}=-x_{1}-x_{2}+\lambda^{2}$ et $y_{3}=\lambda\left(x_{3}-x_{1}\right)-y_{1}$.

Les polynômes de division $\psi_{n}(x, y)$ de la courbe elliptique $E$ sont définis par les relations de récurrence suivantes [4] :

$$
\begin{gathered}
\psi_{0}(x, y)=0 ; \psi_{1}(x, y)=1 ; \psi_{2}(x, y)=2 y \\
\psi_{3}(x, y)=3 x^{4}+6 a x^{2}+12 b x-a^{2} ; \\
\psi_{4}(x, y)=2 y\left(x^{6}+5 a x^{4}+20 b x^{3}-5 a^{2} x^{2}-4 a b x-8 b^{2}-a^{3}\right)
\end{gathered}
$$

et pour $n$ entier, $\psi_{-n}=-\psi_{n}$ puis pour tout $(m, n)$ dans $\mathbb{Z}^{2}$

$$
\psi_{m+n} \psi_{m-n}=\psi_{m+1} \psi_{m-1} \psi_{n}^{2}-\psi_{n+1} \psi_{n-1} \psi_{m}^{2}
$$

Ainsi, $\psi_{2 n+1}=\psi_{n+2} \psi_{n}^{3}-\psi_{n+1}^{3} \psi_{n-1}$ et $\psi_{2 n} \psi_{2}=\psi_{n}\left(\psi_{n+2} \psi_{n-1}^{2}-\psi_{n-2} \psi_{n+1}^{2}\right)$.

\section{Propriétés}

Pour $P=(x, y) \in E\left(\bar{F}_{q}\right)$, on pose $n P=(x(n P), y(n P))=\left(x_{n}, y_{n}\right)$. Les deux résultats qui suivent sont connus mais il n'existe pas dans la littérature de démonstration sans utiliser l'analyse complexe.

Proposition 2. Soit $P=(x, y) \in E\left(\bar{F}_{q}\right)$. On a

$$
\begin{gathered}
x_{n}=x-\frac{\psi_{n-1} \psi_{n+1}}{\psi_{n}^{2}} \\
y_{n}=\frac{\psi_{n+2} \psi_{n-1}^{2}-\psi_{n-2} \psi_{n+1}^{2}}{4 y \psi_{n}^{3}} .
\end{gathered}
$$

Démonstration On a $x_{n+m}=x_{n-m}-\frac{4 y_{n} y_{m}}{\left(x_{n}-x_{m}\right)^{2}}$. Si (1) est vraie au rang $n$ et $m$, alors $x_{n}-x_{m}=\frac{\psi_{m+n} \psi_{m-n}}{\psi_{n}^{2} \psi_{m}^{2}}$. D'autre part, $4 y_{n} y_{m}=\frac{\psi_{2 n} \psi_{2 m}}{\psi_{n}^{4} \psi_{m}^{4}}$ et $\psi_{2 n} \psi_{2 m}=$ $-\psi_{(n-m)+(n+m)} \psi_{(n-m)-(n+m)}=\psi_{n+m-1} \psi_{n+m+1} \psi_{n-m}^{2}-\psi_{n-m+1} \psi_{n-m-1} \psi_{n+m}^{2}$.

Ainsi, si, en plus, (1) est vraie au rang $n-m$, alors elle est vraie au rang $n+m$.

Pour les ordonnées, on utilise l'égalité $x_{n+2}=x_{n}-\frac{4 y_{n+1} y}{\left(x_{n+1}-x\right)^{2}}$.

Une simple récurrence complète la démonstration.

Proposition 3. Soit $P=(x, y) \in E\left(\bar{F}_{q}\right)$. On a

$$
P \in E[r] \Leftrightarrow \psi_{r}(x, y) \equiv 0 \bmod p .
$$


Démonstration • Pour $r=2$, si $p \mid 2 y$, alors $y \equiv-y \bmod p$ ainsi $2 P=(x, y)+$ $(x,-y)=0_{E}$. Réciproquement, si $2 P=O_{E}$, alors $P=-P$ et donc $2 y \equiv 0 \bmod p$.

- Pour $r=2 n$, on utilise l'égalité $\psi_{2 n}=2 y_{n} \psi_{n}^{4}$. Si $p \mid \psi_{2 n}$, alors, par hypothèse de récurrence, le cas $p \mid \psi_{n}$ entraîne $n P=0_{E}$ et si $p \mid y_{n}$, alors $2 n P=n P+n P=$ $n P-n P=0_{E}$. Réciproquement, si $2 n P=O_{E}$, alors $2 y_{n} \equiv 0 \bmod p$.

- Pour $r=2 n+1$, on utilise l'égalité $\psi_{n}^{2} \psi_{n+1}^{2}\left(x_{n}-x_{n+1}\right)=\psi_{2 n+1}$. De $\psi_{2 n+1}=$ $\psi_{n+2} \psi_{n}^{3}-\psi_{n+1}^{3} \psi_{n-1}$, si $p \mid \psi_{n}$, alors $p \mid \psi_{n+1}$ ou $p \mid \psi_{n-1}$ donc $P=0_{E}$. De même si $p \mid \psi_{n+1}$, ainsi $p \mid x_{n}-x_{n+1}$ et on a donc, de l'équation de $(E), p \mid y_{n+1}^{2}-y_{n}^{2}$. Par suite $p \mid y_{n+1}+y_{n}$ d'où $(n+1) P=-n P$. Réciproquement, on a donc $x_{n+1}-x_{n} \equiv 0 \bmod p$ d'où $p \mid \psi_{2 n+1}$.

\section{Périodicité des polynômes de division}

Théorème 1. Soient $\mathbb{F}_{q}$ un corps fini, $E / \mathbb{F}_{q}$ une courbe elliptique et $P \in E\left(\overline{\mathbb{F}}_{q}\right)$ un point d'ordre exact $r \geq 2$. Alors, il existe $w \in \overline{\mathbb{F}}_{q}$, dépendant de $P$, tel que

(1) si $r \geq 3$, alors pour tout $k$ et $n$ de $\mathbb{Z}$,

- si $r=2 m$, on $a$

$$
\psi_{r k+n}(P)=(-1)^{k^{2}} w^{k(n+k m)} \psi_{n}(P) .
$$

- si $r=2 m+1$, on $a$

$$
\psi_{r k+n}(P)=(-1)^{k^{2}} w^{k(2 n+k(2 m+1))} \psi_{n}(P) .
$$

(2) si $r=2$, alors pour tout $k$ de $\mathbb{Z}$

$$
\begin{gathered}
\psi_{4 k+1}(P)=(-1)^{k} \psi_{3}^{k(2 k+1)}, \\
\psi_{4 k+3}(P)=(-1)^{k} \psi_{3}^{(k+1)(2 k+1)} .
\end{gathered}
$$

Remarque Dans sa thèse [2], Shipsey étudie ces suites modulo $p^{2}$. Il serait intéressant d'obtenir une généralisation modulo $p^{k}$ pour $k \in \mathbb{N}$.

Démonstration Les relations sont vraies pour $k=0$.

(1) $\bullet$ Si $r=2 m \geq 3$, alors on pose $w=\frac{\psi_{m+1}}{\psi_{m-1}}$. On suppose d'abord que $k=1$. La relation est donc vraie pour $n=-m+1$. On la démontre par récurrence sur $n$, pour $-m+1 \leq n \leq-1$. Au rang $n+1$, l'égalité $x((2 m+n) P)=$ $x(-n P)$ conduit à l'expression correspondante pour $\psi_{2 m+n+1}$. La relation est évidemment vraie pour $n=-m$ et $n=0$ et donc pour $n$ tel que $-m \leq n \leq 0$. Pour $n$ tel que $-2 m+1 \leq n \leq-m-1$, on pose $-d=r+n$ ainsi $-m+1 \leq$ $d \leq-1$ par suite $\psi_{r+d}(P)=-w^{d+m} \psi_{d}(P)$ et donc $\psi_{-d}=u^{-d-m} \psi_{r+d}(P)$ c'est à dire $\psi_{r+n}(P)=-w^{n+m} \psi_{n}(P)$. De là, on a, pour tous entiers $n$ tels que $-r \leq n \leq 0, \psi_{r+n}(P)=-w^{n+m} \psi_{n}(P)$. Le passage de $n$ à $n+1$ se démontre en exhibant l'expression de $\psi_{r+n+1}$ dans l'égalité $x((2 m+n) P)=x(n P)$ tandis que de $n$ à $n-1$, on exhibe l'expression de $\psi_{r+n-1}$ dans la même égalité. Par suite pour tout $n \in \mathbb{Z}$, on a $\psi_{r+n}=-w^{n+m} \psi_{n}(P)$.

On suppose maintenant la relation de la proposition vraie au rang $k-1$. L'égalité $\psi_{2 k m+1} \psi_{2 k m-3}=-\psi_{1} \psi_{3} \psi_{2 k m-1}^{2}$ conduit à l'expression de $\psi_{2 k m+1}$ $\left(\psi_{2 k m-1}=\psi_{2(k-1) m+2 m-1}\right.$ et similairement pour $\left.\psi_{2 k m-3}\right)$ et $\psi_{2 k m+2} \psi_{2 k m-2}$ $=\psi_{2 k m+1} \psi_{2 k m-1} \psi_{2}^{2}$ conduit à celle de $\psi_{2 k m+2}$. On établit une récurrence sur 
$n$ à partir de l'égalité $x((2 k m+n-1) P)=x((n-1) P)$ qui donne l'expression de $\chi_{n}=\frac{\psi_{k r+n}(P)}{\psi_{n}(P)}$ en fonction de $\chi_{n-1}$ et $\chi_{n-2}$. La récurrence descendante, pour obtenir la relation pour les entiers $k$ négatifs, s'obtient à partir de $\psi_{2(k-1) m+n}(P)=\psi_{2 k m+n-2 m}(P)$.

- Si $r=2 m+1 \geq 3$, alors on procède de la même manière avec $w=\frac{\psi_{m+1}}{\psi_{m}}$.

(2) Une récurrence, pour le cas $r=2$, en utilisant $\psi_{2 n+1}=\psi_{n+2} \psi_{n}^{3}-\psi_{n+1} \psi_{n-1}$ conduit facilement au résultat.

\section{Exemple}

\begin{tabular}{|c|c|c|c|c|c|c|c|c|c|c|c|}
\hline \hline$a$ & $b$ & $p$ & $P$ & $r$ & $m$ & $w$ & $k$ & $n$ & $k r+n$ & $\psi_{k r+n}$ & $(-1)^{k^{2}} w^{-} \psi_{n}$ \\
\hline 1 & 1 & 53 & $(32,43)$ & 29 & 14 & 1 & 2 & 3 & 61 & 27 & 27 \\
\hline & & & & & & & 7 & 9 & 212 & 39 & 39 \\
\hline & & & & & & & 27 & 21 & 804 & 46 & 46 \\
\hline 3 & 2 & 101 & $(2,4)$ & 20 & 10 & 90 & 2 & 21 & 59 & 37 & 37 \\
\hline & & & & & & & 21 & 12 & 432 & 28 & 28 \\
\hline & & & & & & & 57 & 73 & 1213 & 63 & 63 \\
\hline \hline
\end{tabular}

Corollaire 1. Si $P \in E\left(\overline{\mathbb{F}}_{q}\right)$ un point d'ordre exact égal à $r$, alors la suite $\left(\psi_{n}(P)\right)_{n \in \mathbb{N}}$ est une suite périodique de période ord $(P) t$ avec $t \mid q-1$ si ord $(P) \geq 3$ et $t \mid 2 q-2$ si $\operatorname{ord}(P)=2$.

Démonstration C'est une simple conséquence de l'expression de l'exposant de $w$ lorsque $r \geq 3$ et de $\psi_{3}$ lorsque $r=2$ dans la proposition précédente. D'ailleurs, la période s'exprime en fonction de $\operatorname{ord}(P) \operatorname{ord}(w)$ suivant les parités respectives.

Exemple Soit la courbe elliptique d'équation $y^{2}=x^{3}+x+1$ sur $\mathbb{F}_{p}$.

\begin{tabular}{|c|c|c|c|c|c|c|c|}
\hline \hline$p$ & $P$ & $r=\operatorname{ord}(P)$ & $m$ & $w$ & ord $(w)$ & période & ord $(w) \operatorname{ord}(P)$ \\
\hline 23 & $(18,20)$ & 28 & 14 & 10 & 22 & 616 & 616 \\
\hline 23 & $(12,19)$ & 14 & 7 & 16 & 11 & 308 & 154 \\
\hline 23 & $(5,4)$ & 7 & 3 & 9 & 11 & 154 & 77 \\
\hline 53 & $(8,16)$ & 29 & 14 & 22 & 52 & 754 & 1508 \\
\hline 61 & $(46,37)$ & 25 & 12 & 27 & 10 & 125 & 250 \\
\hline 61 & $(42,57)$ & 25 & 12 & 9 & 5 & 250 & 125 \\
\hline \hline
\end{tabular}

\section{Remerciements}

L'auteur tient à remercier le rédacteur de MRL pour ces remarques constructives.

\section{Références}

[1] M. Ayads, Périodicité (mod q) des suites elliptiques et points $S$-entiers sur les courbes elliptiques, Annales de l'institut de Fourier, 43(3) (1993), 585-618.

[2] R. Shipsey, Elliptic divisibility sequences, Ph. D Thesis, Goldsmith's College (University of London), 2000.

[3] J. H. Silverman, p-adic properties of division polynomials and elliptic divisibility sequences, Mathematische Annalen, 332(2)(2005), 443-471.

[4] 1986.

[5] M. Ward, Memoir on elliptic divisibility sequences, Amer. J. Math 70 (1948), 31-74. 
[6] (1948), 941-946.

ESIEE-Amiens, Ecole Supérieure d'Ingénieurs en Electronique et Electrotechnique, 14 QUAI DE LA SOMME-BP100, F-80082 Amiens CedeX2

E-mail address: dewaghe@esiee-amiens.fr 